\title{
Cannabinoid Receptor Agonist WIN55, 212-2 Adjusts Lipid Metabolism in a Rat Model of Cardiac Arrest
}

\author{
Yan Xiao, MD, PhD, ${ }^{1,2, *}$ Daniel Contaifer, BS, ${ }^{3, *}$ Weiping Huang, MD, ${ }^{2}$ Jin Yang, MD, PhD, ${ }^{2}$ Zhangle Hu, MD, ${ }^{2}$ \\ Qinyue Guo, MD, ${ }^{2}$ Jennifer Bradley, MS, ${ }^{2}$ Mary Ann Peberdy, MD, ${ }^{2,4,5}$ Joseph P. Ornato, MD, 2,6 \\ Dayanjan S. Wijesinghe, PhD, $3,7-9, \dagger$ and Wanchun Tang, $\mathrm{MD}^{2,6, \dagger}$
}

The objective of this study was to investigate the effects of pharmacologically induced hypothermia with WIN55, 212-2 (WIN)on postresuscitation myocardial function, microcirculation, and metabolism-specific lipids in a rat cardiac arrest (CA) model. Ventricular fibrillation was electrically induced and untreated for 6 minutes in 24 Sprague-Dawley rats weighing 450-550 g. Cardiopulmonary resuscitation including chest compression and mechanical ventilation was then initiated and continued for 8 minutes, followed by defibrillation. At 5 minutes after restoration of spontaneous circulation (ROSC), animals were randomized into four groups: (1) normothermia with vehicle (NT); (2) physical hypothermia with vehicle (PH); (3) WIN55, 212-2 with normothermia (WN); and (4) WIN55, 212-2 with hypothermia (WH). For groups of WN and WH, WIN was administered by continuous intravenous infusion with a syringe pump for 4 hours. PH started at 5 minutes after resuscitation. NT maintained core temperature at $37^{\circ} \mathrm{C} \pm 0.2^{\circ} \mathrm{C}$ with the aid of a heating blanket. Hypothermia groups maintained temperature at $33^{\circ} \mathrm{C} \pm 0.5^{\circ} \mathrm{C}$ for 4 hours after ROSC. There was a significant improvement in myocardial function as measured by ejection fraction, cardiac output, and myocardial performance index in animals treated with WH and $\mathrm{PH}$ beginning at 1 hour after start of infusion. In the $\mathrm{WH}$ and $\mathrm{PH}$ groups, buccal microcirculation was significantly improved compared with NT and WN. Plasma at pre-CA and ROSC 4 hours was harvested for lipid metabolism. The WH group appeared to be closer to baseline than the other groups in lipid metabolism. lysophosphatidylcholine (LPC) 18:2, free fatty acid (FFA) 22:6, and ceramide (CER) (24:0) changed significantly among the lipidomic data compared with NT $(p<0.05)$. Postresuscitation hypothermia improved myocardial function and microcirculation. WH-mediated lipid metabolism had the best metabolic outcome to bring back the animals to normal metabolism, which may be protective to improve outcomes of CA. LPC 18:2, FFA 22:6, and CER (24:0) may be important predictors of outcomes of CA.

Keywords: cannabinoid receptor, cardiopulmonary resuscitation, buccal microcirculation, shivering, lipid metabolism

\section{Introduction}

B ASED ON THE FAVORABLE outcome of both clinical (Bernard et al., 2002; Hypothermia after Cardiac Arrest Study Group, 2002) and laboratory studies, the American Heart Association recommended the use of therapeutic hypothermia for routine management of cardiac arrest (CA) patients after initial successful resuscitation (Callaway et al.,
2015). Unfortunately, currently available therapeutic hypothermia techniques are essentially based on the physical cooling method. These techniques are less effective and mostly limited for in-hospital use, which depend on the instrument. Current methods such as cold blankets, ice pad, and chilled intravenous fluids are easy to implement but are limited by effectiveness and side-effects (Lampe et al., 2007). Shivering resulting from physical cooling occurs frequently (84.6\%) (MacLaren et al.,

\footnotetext{
${ }^{1}$ Department of Emergency Medicine, The Second Affiliated Hospital of Soochow University, Soochow, China.

${ }^{2}$ Weil Institute of Emergency and Critical Care Research, Virginia Commonwealth University, Richmond, Virginia, USA.

${ }^{3}$ School of Pharmacy, Virginia Commonwealth University, Richmond, Virginia, USA.

Departments of ${ }^{4}$ Internal Medicine and ${ }^{5}$ Emergency Medicine, Virginia Commonwealth University Health System, Richmond, Virginia, USA.

${ }^{6}$ Department of Emergency Medicine, Virginia Commonwealth University, Richmond, Virginia, USA.

${ }^{7}$ Institute for Structural Biology, Drug Discovery and Development, Virginia Commonwealth University, Richmond, Virginia, USA.

${ }^{8}$ Da Vinci Center and ${ }^{9}$ Department of Pharmacotherapy and Outcomes Sciences, School of Pharmacy, Virginia Commonwealth University School of Medicine, Richmond, Virginia, USA.

*Co-first authors, equal contributions.

${ }^{\dagger}$ Co-senior authors, equal contributions.
} 
2014). It not only increases the difficulty of achieving and maintaining target temperature, but early neurologic examination is compromised by the associated requirement for sedation and paralysis as well (Pollock et al., 2016).

Cannabinoid receptors (CBs) located in the preoptic anterior hypothalamus play a major role in thermoregulation by maintaining a constant "core" temperature (Yoshida et al., 2009). The nonselective CB agonist, WIN 55, 212-2, also plays an important role in thermoregulation related to the activation of central $\mathrm{CB} 1$ receptors. Our previous studies have demonstrated that WIN55, 212-2-induced mild hypothermia improved the outcome of cardiopulmonary resuscitation (CPR) in a rat model of CA (Sun et al., 2010; Ma et al., 2014). This hypothermic effect was completely blocked by a CB1 but not a CB2 antagonist (Weng et al., 2012). However, the mechanism has not been elucidated.

Previous studies have shown that damage in the brain is responsible for high mortality of patients after initial resuscitation (Mongardon et al., 2011; Stub et al., 2011). Successful treatment of patients depends first on restoration of spontaneous circulation (ROSC), and second on normalization of impaired metabolic pathways and homeostasis. A major component of plasma membrane is lipid. These molecules have a wide range of functions, including cellular structure, metabolism, and signaling (Murakami, 2011). There is a group of lipid species that are distinguished by their involvement in neurotransmission, primarily acting as neuromodulators of other systems. These specific lipids reach their highest degree of specialization and diversity in the central nervous system and could be called neurolipids in the same way that neuronal-specific proteins are called neuropeptides. Some lipids that act as signaling molecules originate from phospholipid (PL) precursors present in the plasma membrane and are synthesized on demand. Studies have suggested that the decomposition of membrane PLs is an important event in ischemia (Rink and Khanna, 2011).

Preliminary mass spectrometry-based lipidomic and metabolomic analysis in humans undergoing therapeutic hypothermia has allowed us to identify metabolic products that are generated in response to hypothermia. We hypothesize that improved outcomes after WIN55, 212-2 administration not only resulted from hypothermia but also from alterations to the metabolism of specific lipids after CA.

\section{Materials and Methods}

This study was approved by the Institutional Animal Care and Use Committee of Virginia Commonwealth University. Animals were handled in accordance with the Guide for the Care and Use of Laboratory Animals published by the National Institute of Health.

\section{Animal preparation}

Male Sprague-Dawley rats, 6-10 months of age weighing between 450 and $550 \mathrm{~g}$, were supplied by a single-source breeder (Envigo, Frederick, Maryland). After induction of anesthesia with inhalation of $100 \% \mathrm{CO}_{2}$ for 30 seconds, the animals were anesthetized by intraperitoneal injection of pentobarbital $(45 \mathrm{mg} / \mathrm{kg})$. Additional doses $(10 \mathrm{mg} / \mathrm{kg})$ were administered at hourly intervals or when required to maintain anesthesia. Depth of anesthesia was monitored by tail pinch. The trachea was orally intubated with a 14-gauge cannula mounted on a blunt needle (Abbocath-T; Abbott Hospital
Products Division, North Chicago, IL) with a 145-degree angled tip. End-tidal $\mathrm{CO}_{2}\left(\mathrm{ETCO}_{2}\right)$ was continuously monitored with a side-stream infrared $\mathrm{CO}_{2}$ analyzer (Capstar-100 Carbon Dioxide Analyzer; CWE, Ardmore, PA).

A conventional lead II EKG was continuously monitored. For blood pressure measurements within the descending aorta and right atrium and drug administration, polyethylene catheters (PE-50; Becton Dickinson, Sparks, MD) were advanced into the descending aorta from the left femoral artery, the right atrium from the left external jugular vein, and the inferior vena cava from the right femoral vein, respectively. A thermocouple microprobe (IT-18; Physitemp Instruments, Inc., NJ) was inserted into the descending aorta from the right femoral artery for the measurement of blood temperature. A 3-F catheter (Model C-PMS-301J; Cook Critical Care, Bloomington, IN) was advanced through the right external jugular vein into the right atrium. A precurved guide wire supplied with the catheter was then advanced through the catheter into the right ventricle to induce ventricular fibrillation (VF), as confirmed by an endocardial electrocardiograph. All catheters were flushed intermittently with saline containing $2.5 \mathrm{IU} / \mathrm{mL}$ of crystalline bovine heparin. The core temperature (aortic blood temperature) was maintained at $37^{\circ} \mathrm{C} \pm 0.5^{\circ} \mathrm{C}$ by a heating blanket.

\section{Experimental procedures}

Twenty-four rats were randomized into four groups (each group $n=6$ ): Group 1: normothermia with vehicle (NT); Group 2: physical hypothermia with vehicle (PH); Group 3: WIN55, 212-2 with normothermia (WN); and Group 4: WIN55, 212-2 with hypothermia (WH). Blood temperature was stabilized at $37^{\circ} \mathrm{C} \pm 0.2^{\circ} \mathrm{C}$ for 30 minutes before resuscitation with use of a heating blanket for all groups. Fifteen minutes before induction of VF, baseline measurements and echocardiography were obtained. Mechanical ventilation was established at a tidal volume of $0.60 \mathrm{~mL} / 100 \mathrm{~g}$ of body weight, a frequency of 100 breaths/min, and an inspired $\mathrm{O}_{2}$ fraction $\left(\mathrm{FiO}_{2}\right)$ of 0.21 . VF was then induced through a guide wire advanced from the right jugular vein into the right ventricle. A progressive increase in $60 \mathrm{~Hz}$ current to a maximum of $3.5 \mathrm{~mA}$ was then delivered to the right ventricular endocardium. The current flow was continued for 3 minutes to prevent spontaneous defibrillation. Mechanical ventilation was discontinued after onset of VF.

After 6 minutes of untreated VF, precordial compressions, together with mechanical ventilation (tidal volume $0.60 \mathrm{~mL} / 100 \mathrm{~g}$ body weight, frequency 100 breaths $/ \mathrm{min}, \mathrm{FiO}_{2}$ 1.0), were initiated using a pneumatically driven mechanical chest compressor. Precordial chest compressions were maintained at a rate of $200 / \mathrm{min}$ and synchronized to provide a compression/ventilation ratio of 2:1 with equal compressionrelaxation for a duration of 8 minutes. Defibrillation was attempted with up to three 4-J counter shocks after 8 minutes of CPR. ROSC was defined as the return of supraventricular rhythm with a mean aortic pressure $>50 \mathrm{mmHg}$ for 5 minutes. If ROSC was not achieved after the first defibrillation attempt, a 30-second interval of CPR was performed before the next defibrillation attempt (up to three attempts). After ROSC, a $\mathrm{FiO} 2$ of 1.0 was continued for 1 hour, adjusted to 0.5 for the second hour and 0.21 for the last 2 hours.

At 5 minutes after ROSC, either WIN55, 212-2 $(1 \mathrm{mg} / \mathrm{kg} / \mathrm{h})$ or vehicle ( $2 \%$ Tween-80 in $0.9 \% \mathrm{NaCl}$ solution) was administered by continuous intravenous infusion with a 
syringe pump (Genie Touch; Kent Scientific Corporation) for 4 hours at a rate of $1.4 \mathrm{~mL} / \mathrm{kg} / \mathrm{h}$ (Weng et al., 2012). In Group 2, physical hypothermia started at 5 minutes after resuscitation with a cooling pad once blood temperature reached $34^{\circ} \mathrm{C}$ (target temperature $32-34^{\circ} \mathrm{C}$ ). In Group 3, temperature was maintained at $37^{\circ} \mathrm{C} \pm 0.2^{\circ} \mathrm{C}$ with the aid of a heating blanket. External warming was maintained consistent in all groups to guarantee Group 4 hypothermia was solely due to drug (Fig. 1). At 4 hours after ROSC, $1 \mathrm{~mL}$ of blood was withdrawn, and animals were euthanized with Euthasol $(150 \mathrm{mg} / \mathrm{kg})$.

\section{Measurements}

Hemodynamics and other parameters. Electrocardiogram, aortic and right atrial pressures, blood temperature, $\mathrm{ETCO}_{2}$ and electromyography (EMG) values were continuously recorded on a personal computer-based data-acquisition system supported by WINDAQ software (DATAQ, Akron, OH). Coronary perfusion pressure (CPP) was calculated as the difference between aortic and time-coincident right atrial pressures measured at the end of each minute of precordial compression. The start time, intensity, and duration time of shivering according to changes of EMG signal were recorded.

At baseline, 1, 2, 3, and 4 hours after ROSC, ejection fraction $(\mathrm{EF})$, cardiac output $(\mathrm{CO})$, and myocardial performance index (MPI) were measured by echocardiography (HD11XE; Philips Medical Systems, Eindhoven, Netherlands) with a $12.5 \mathrm{~Hz}$ transducer. $\mathrm{CO}$ and $\mathrm{EF}$ are adopted to estimate the myocardial contractility; MPI is adopted to estimate left ventricular diastolic function. The MPI, which combines time intervals related to systolic and diastolic functions reflecting the global cardiac function, is also calculated using the formula $(a-b) / b$, where $\mathrm{a}=$ mitral closure-to-opening interval (time interval from cessation to onset of mitral inflow) and $b=E T$ (aortic flow ejection time, obtained at the left ventricle outflow tract).

Buccal microcirculation was measured at baseline, 1, 2, 3 , and 4 hours after ROSC with the aid of a side-stream dark-field imaging device (Micro Scan; Micro Vision Medical, Inc., Amsterdam, the Netherlands) that has a $5 \times$ imaging objective, resulting in an on-screen magnification of $276 \times$. Three discrete fields for each were captured with the intention to minimize motion artifacts. Microvascular images were recorded on a DVD recorder (DMR-EZ47V; Panasonic AVC Networks, Dalian, China). Microvascular flow index (MFI) was quantitated by the method of Salgado et al. (2011). The image was divided into four quadrants, and predominant type of flow was assessed in the small vessels of each quadrant, which were $<20 \mu \mathrm{m}$ in diameter. The MFI score represents the average values of the four quadrants. Perfused vessel density (PVD) is measured based on the method of De Backer et al. (2007).

\section{Sample processing for mass spectrometry analysis}

Blood was collected from all animals at baseline and postresuscitation 4 hours $(\mathrm{PR} 4 \mathrm{H})$. Blood was processed to obtain plasma by collection into $1.5 \mathrm{~mL}$ Eppendorf tubes containing $5 \mu \mathrm{L}$ of $0.5 \mathrm{M}$ EDTA. Samples were mixed and stored on ice. They were then spun down at $1500 \mathrm{~g}$ for 15 minutes. The supernatant was aliquoted into cryostorage vials and snap frozen with liquid nitrogen. The samples thus processed were stored at $-80^{\circ} \mathrm{C}$ until lipidomic analysis.

Samples were prepared for analysis using an HILIC-based UPLC ESI-MS/MS method. Fifty microliters of plasma was added to $750 \mu \mathrm{L}$ of methyl-tertiary butyl ether (MTBE), containing $20 \mu \mathrm{L}$ of SPLASH internal standards (SPLASH LIPIDOMIX Mass Spec Standard-Avanti 330707) and $160 \mu \mathrm{L}$ of water. After centrifugation for 2 minutes at $12,300 \mathrm{rpm}, 350 \mu \mathrm{L}$ of supernatant was transferred to autosampler vials and dried under vacuum. Dried extracts were resuspended using $110 \mu \mathrm{L}$ of a methanol:toluene (10:1, v/v) mixture containing CUDA (12-[[(cyclohexylamino) carbonyl] amino]-dodecanoic acid) at a final concentration of $50 \mathrm{ng} / \mathrm{mL}$.

Samples were analyzed on a QTRAP 6500+, with Shimadzu Nexera UPLC. Analytes were separated on a Waters BEH HILIC $1.7 \mu \mathrm{m} 2.1 \times 150 \mathrm{~mm}$ column (column temperature $=30^{\circ} \mathrm{C}$ ). Mobile phase $\mathrm{A}: 10 \mathrm{mM}$ ammonium acetate (pH 8) in $95 \%$ ACN (acetonitrile). Mobile phase B: $10 \mathrm{mM}$ ammonium acetate $(\mathrm{pH})$ in $50 \%$ ACN. Gradient $(\mathrm{B} \%)$ ramped from 0.1 to 20 in 10 minutes; rose to 98 at 11 minutes, was then kept for 2 minutes, then dropped back to 0.1 , and was then maintained for 3 minutes.

\section{Statistical analysis}

Statistical analysis was performed with SPSS 17.0 software (SPSS, Inc., Chicago, IL). After confirmation of normal distribution with the Kolmogorov-Smirnov test, all variables were compared either using parametric tests (analysis of variance [ANOVA]) or nonparametric tests (Kruskal-Wallis test). For measurements among groups, ANOVA and Scheffe multiple-comparison techniques were employed. Comparisons between time-based measurements within each group were performed with ANOVA repeated measures. All data are reported as mean $\pm S D$.

Lipids were used to find predictors of group allocation to determine the metabolic pattern compared with baseline values. Stepwise regularized linear discriminant analysis was used as a statistical approach to select these predictors. Lipids were accepted in the model until minimum percentage of misclassification was achieved. Lipids were assumed as normally distributed, and ANOVA with Tukey's post hoc was used to determine statistical significance with $p<0.05$ and false discovery rate $=0.05$ as selection criteria. Statistical validation was performed with permutation test based on partial least squares-discriminant analysis. The validation model calculated the ratio of the between sum of the squares and the within sum of squares $(\mathrm{B} / \mathrm{W})$ for the class assignment prediction of 2000 permuted models. The test estimates the statistical significance of the original class discrimination calculating if its $\mathrm{B} / \mathrm{W}$ ratio is not a part of the distribution of the permuted class assignments. Random Forest modeling was used to reveal important lipids to predict the group's separation.

\section{Results}

Twenty-eight rats were used for this study. Four rats were not resuscitated (one, technical failure; three, resuscitation failure). Twenty-four rats were resuscitated successfully and randomized into four groups by envelope blinding to be included in the study. There were no differences in baseline hemodynamics, CPP (Table 1), myocardial function (CO, EF, MPI), or buccal microcirculation among the four groups. Blood temperature in $\mathrm{WH}$ group decreased progressively from $36.8^{\circ} \mathrm{C} \pm 0.2^{\circ} \mathrm{C}$ to $35.1^{\circ} \mathrm{C} \pm 0.5^{\circ} \mathrm{C}$ during the first hour of infusion and reached $33.3^{\circ} \mathrm{C} \pm 0.3^{\circ} \mathrm{C}$ at 4 hours after infusion. In our study, the duration of reduced blood temperatures did not have a significant 
Table 1. Characteristics in Body Weight, Coronary Perfusion Pressure, Number of Defibrillations, Heart Rate, Mean Arterial Pressure, and PetCO 2

\begin{tabular}{lccrr}
\hline Group & Normothermia & Physical hypothermia & Win55+normothermia & Win55+hypothermia \\
\hline Weight (g) & $512 \pm 8.7$ & $510 \pm 6.2$ & $501.3 \pm 7.9$ & $507 \pm 4.6$ \\
CPP in PC1 (mmHg) & $39.3 \pm 4.4$ & $36.8 \pm 7.3$ & $36.17 \pm 4.4$ & $36.5 \pm 5.0$ \\
CPP in PC8 (mmHg) & $30.33 \pm 1.2$ & $26.83 \pm 3.3$ & $28.3 \pm 2.1$ & $28.8 \pm 4.5$ \\
No. of defibrillations & $1.7 \pm 0.8$ & $1.3 \pm 0.5$ & $2.3 \pm 2.1$ & $2.8 \pm 0.9$ \\
HR (bpm) & $361 \pm 23$ & $369 \pm 15$ & $366 \pm 17$ & $374 \pm 39$ \\
Before VF & $360 \pm 15$ & $322 \pm 18^{\mathrm{a}, \mathrm{b}}$ & $283 \pm 28^{\mathrm{a}, \mathrm{b}}$ & $225 \pm 13^{\mathrm{a}}$ \\
PR2H & $361 \pm 27$ & $313 \pm 24^{\mathrm{a}, \mathrm{b}}$ & $323 \pm 33^{\mathrm{a}, \mathrm{b}}$ & $255 \pm 18^{\mathrm{a}}$ \\
PR4H & & & $141 \pm 9$ & \\
MAP (mmHg) & $142 \pm 5$ & $139 \pm 12$ & $102 \pm 18^{\mathrm{a}}$ & $96 \pm 13^{\mathrm{a}}$ \\
Before VF & $111 \pm 9$ & $115 \pm 8^{\mathrm{b}}$ & & $92 \pm 13^{\mathrm{a}}$ \\
PR2H & $117 \pm 9$ & $115 \pm 3^{\mathrm{b}}$ & $35.9 \pm 4.7$ & $37.1 \pm 3.6$ \\
PR4H & & $32.2 \pm 5.1$ & $37.9 \pm 3.6^{\mathrm{a}}$ \\
PetCO (mmH) & $33.6 \pm 1.8$ & $33.1 \pm 3.0$ & $31.4 \pm 4.4^{\mathrm{b}}$ & $37.8 \pm 2.7^{\mathrm{a}}$ \\
Before VF & $29.8 \pm 2.6$ & $30.5 \pm 2.1$ & & \\
PR2H & $27.7 \pm 2.2$ & $33 \pm 3.0^{\mathrm{a}}$ & & \\
PR4H & & & & \\
\hline
\end{tabular}

${ }^{\mathrm{a}} p<0.05$ versus normothermia.

${ }^{\mathrm{b}} p<0.05$ versus win $55+$ hypo.

CPP, coronary perfusion pressure; HR, heart rate; PC1, 1 minute after precardiac compressure; PC8, 8 minutes after precordial compression; $\mathrm{PetCO}_{2}$, pressure of end-tidal $\mathrm{CO}_{2}$; PR, postresuscitation; MAP, mean arterial pressure; VF, ventricular fibrillation.

difference between WH and $\mathrm{PH}$, which had similar temperature drop rate. Due to the use of a heating blanket, there was no significant reduction in blood temperature in animals with $\mathrm{WN}$, which was similar to NT (Fig. 2).

Shivering was monitored by EMG and was only observed in the physical hypothermia group. It was significant in the physical hypothermia group compared with all other groups. Shivering in physical hypothermia started at $134.67 \pm 17.45$ minutes and ended at $186.67 \pm 32.35$ minutes after ROSC. Duration of shivering was $52 \pm 43.68$ minutes (Fig. 3).

Mean arterial pressure (MAP) and heart rate (HR) in WH and WN decreased significantly compared with NT at PR1H, $\mathrm{PR} 2 \mathrm{H}, \mathrm{PR} 3 \mathrm{H}$, and $\mathrm{PR} 4 \mathrm{H}$, while $\mathrm{ETCO}_{2}$ in $\mathrm{WH}$ increased significantly compared with NT (Fig. 4). Myocardial function, as measured by CO, EF, and MPI, was significantly impaired in all animals after successful resuscitation compared with baseline values. There was a significant improvement in myocardial function in animals treated with WH beginning at 1 hour after start of infusion, which is the same with physical hypothermia. Compared with NT and WN, we also found that WH was significantly improved at PR4H compared with the $\mathrm{PH}$ group with respect to myocardial function (Fig. 4).

In the NT group, buccal microcirculatory blood flow presented at a low-density and inadequate perfused state after resuscitation, which was also observed in WN. However, in the WH and $\mathrm{PH}$ groups, there were significant improvements in PVD and MFI compared with NT and WN at PR4H (Fig. 5). To understand the accompanying changes to the lipidome, baseline values and four different treatments were analyzed to obtain a linear discriminant model that best represented the group's separation. Baseline data (Base, $n=24$ ) from each group were combined together in a unique group representing a common start point for each of the four treatments. The analysis selected 14 lipids as predictors of group's membership for the six animals used in four treatment groups. Group's separation based on canonical components showed that WH was the closest to baseline (Base), indicating that this treatment had the best metabolic outcome to bring back the animals to a normal metabolism (Fig. 6A, B).

The model with 14 predictors was cross-validated with permutation test. This test investigated whether the modeling conditions might be finding "chance correlation" between predictors and groups or overfitting the data. The test added information decisive for the existence of the model, showing that the observed statistics were significantly different from random permutation (Fig. 7A). Mean decreased accuracy showed that lysophosphatidylcholine (LPC) 18:2, free fatty acid (FFA) 22:6, and ceramide (CER) 24:0 were the most

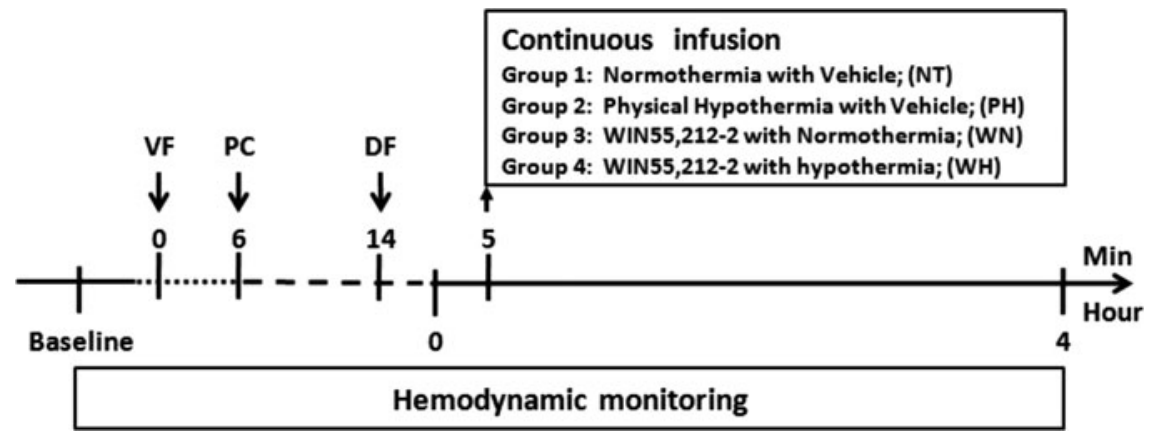

FIG. 1. Experimental outline. DF, defibrillation; PC, precordial compression; VF, ventricular fibrillation. 


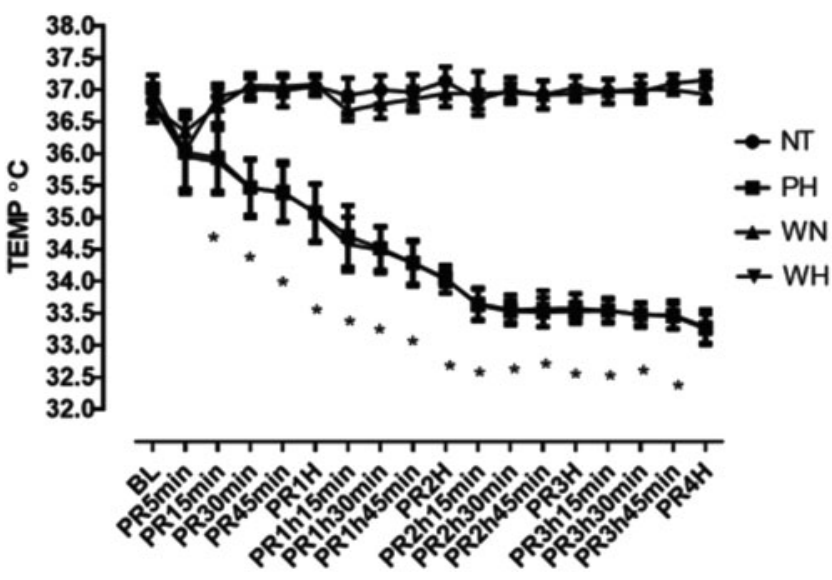

FIG. 2. Temperature drop in each group, $\mathrm{PH}$ and $\mathrm{WH}$ groups reach $34^{\circ} \mathrm{C}$ at PR2 10 minutes. Significant differences are observed in both hypothermia groups (WH and $\mathrm{PH}$ ) compared with both normothermia groups (NT and WN) $\left({ }^{*} p<0.05\right)$. NT, normothermia with vehicle; $\mathrm{PH}$, physical hypothermia with vehicle; WH, WIN55, 212-2 with hypothermia; WN, WIN55, 212-2 with normothermia.

important variables in the model (Fig. 7B). The Random Forest classification method is highly accurate when dealing with large datasets, but can lose power and tend to over fit in small studies. Therefore, it was used here only with the aim of highlighting the most important variable. Nevertheless, the method showed a misclassification error of $15 \%$, all due to the proximity of WH to baseline. Three important lipids to predict the models are statistically different from baseline Fig. 7C), and are specific markers of WH (LPC 18:2), PH (FA 22:6), and NT (Cer 24:0).

\section{Discussion}

Our study demonstrated that the nonselective CB receptor agonist, WIN55, 212-2, induced mild hypothermia after resuscitation, which resulted in significantly improved postresuscitation microcirculation, myocardial function, and regulation of lipid metabolism homeostasis in a rat model of CA. We also demonstrated that there was no shivering during pharmacologically induced hypothermia by WIN 55 , 212-2. We further demonstrated that WIN55, 212-2 provided a powerful protection of lipid metabolism during CA and resuscitation.

Therapeutic hypothermia has been demonstrated as a lifesaving therapy after resuscitation from $\mathrm{CA}$. It has been recommended as a standard of care by the American Heart Association CPR guidelines since 2005. However, the widespread use of this life-saving therapy is limited because all currently available cooling techniques are based on the physical cooling principle. While the adoption of this therapy has led to improved outcomes from CA at multiple hospitals worldwide, the time from arrest to target temperature achievement is still on the order of hours (Fink et al., 2007; Gaieski et al., 2009). Shivering, a major disadvantage of physical cooling, increases heat production two- to fivefold, and is activated when behavioral compensations and maximal vasoconstriction are insufficient to maintain core temperature (Giesbrecht et al., 1994).

Shivering is an involuntary muscular activity that enhances heat production in an attempt to restore homeostasis (De Witte and Sessler, 2002). Available data suggest that the shivering threshold is $1^{\circ} \mathrm{C}$ lower than vasoconstriction threshold (Lopez et al., 1994). When the shivering threshold is reached, motor neurons are recruited in sequence and with increasing size, as hypothermia persists (De Witte and Sessler, 2002). The end result of this autoregulatory process is a
FIG. 3. Shivering duration time among all groups and an example of EMG recording on shivering. $* p<0.05$ versus NT; ${ }^{*} p<0.05$ versus WH. EMG, electromyography.
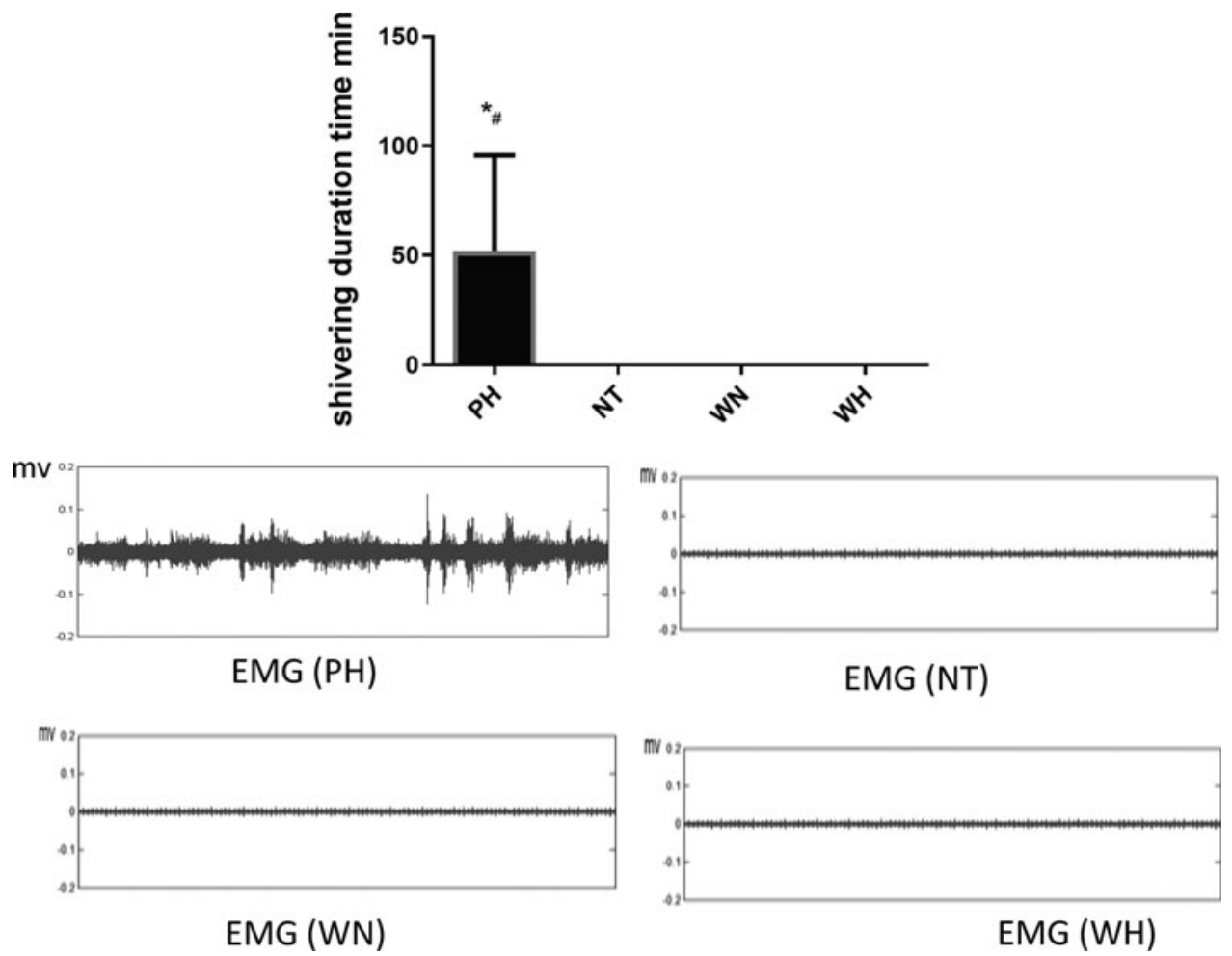

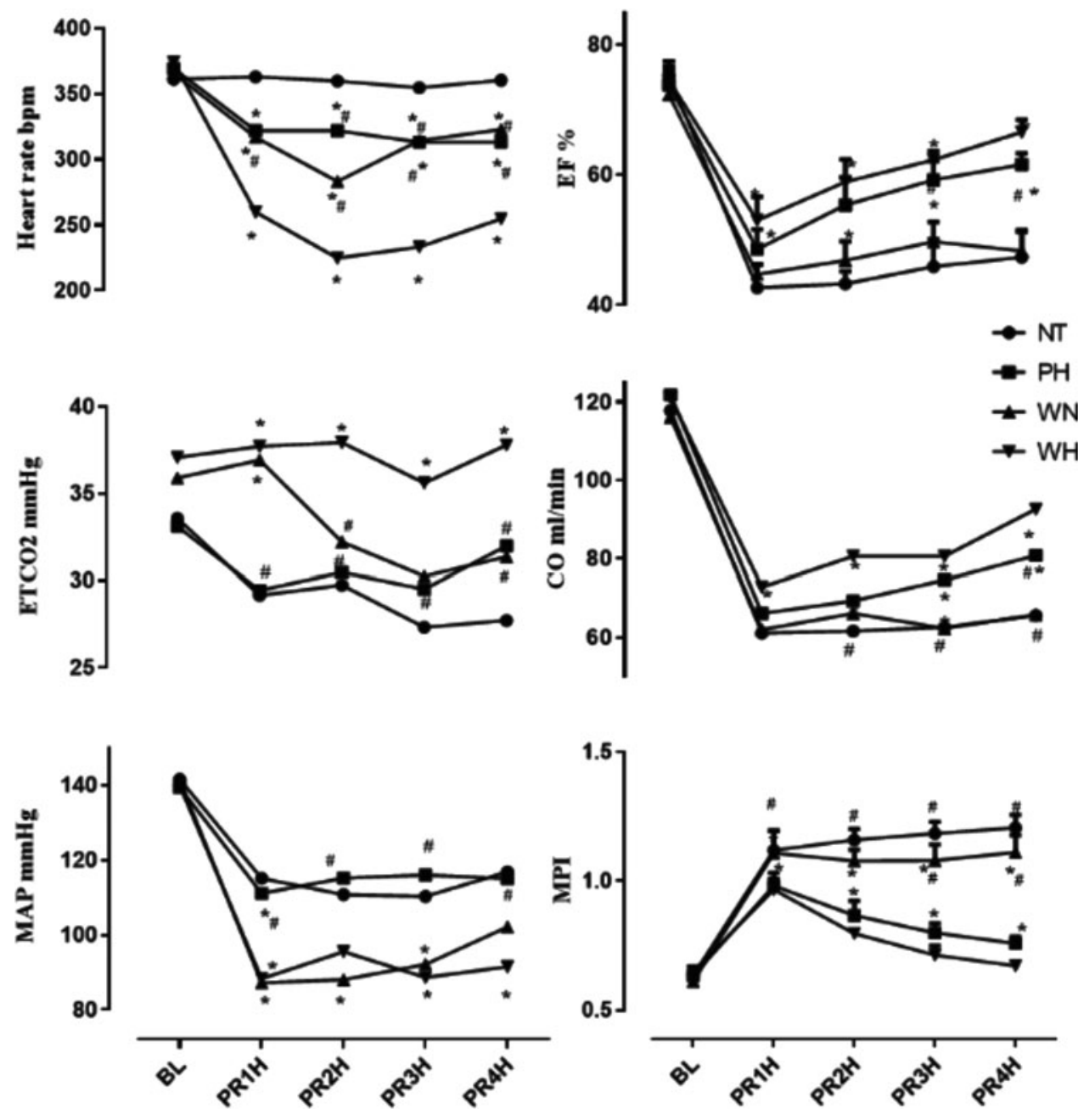

FIG. 4. MAP and HR in WH and WN decreased significantly compared with NT at $\mathrm{PR} 1 \mathrm{H}, \mathrm{PR} 2 \mathrm{H}, \mathrm{PR} 3 \mathrm{H}$, and $\mathrm{PR} 4 \mathrm{H}$, while $\mathrm{ETCO}_{2}$ in $\mathrm{WH}$ increased significantly compared with NT; there was a significant improvement in myocardial function in animals treated with WH beginning at 1 hour after start of infusion, which is the same with $\mathrm{PH}$. WH was significantly improved at PR4H compared with the $\mathrm{PH}$ group. ${ }^{*} p<0.05$ versus NT; $\#_{p}<0.05$ versus $\mathrm{WH}$. BL, baseline; $\mathrm{ETCO}_{2}$, end-tidal $\mathrm{CO}_{2}$; HR, heart rate; MAP, mean arterial pressure; PR, postresuscitation.
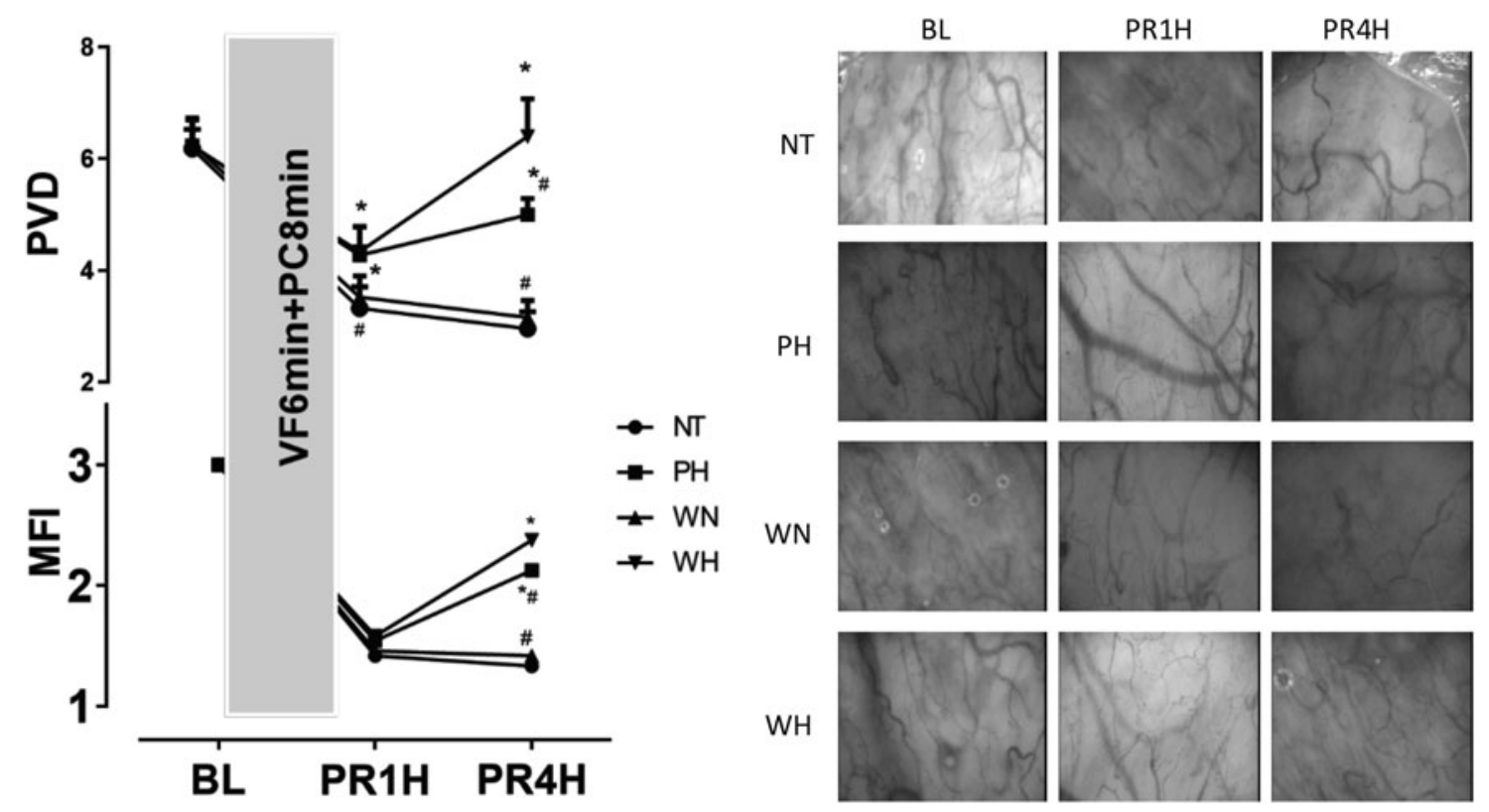

FIG. 5. In the WH and PH groups, there were significant improvements in PVD and MFI compared with NT and WN, ${ }^{*} p<0.05$ versus NT; ${ }^{\#} p<0.05$ versus WH. MFI, microvascular flow index; PVD, perfused vessel density. 
FIG. 6. Regularized linear discriminant analysis shows separation of baseline against normothermia treatment and hypothermia treatments. WH treatment appears to be closer to baseline than the other groups $(\mathbf{A}, \mathbf{B})$. The + sign represents the position of the group mean in the plot. The circle represents $95 \%$ confidence region to contain the mean. The discriminant separation of groups in the model was maximized with inclusion of all 14 lipids with $1.85 \%$ chance of misclassification.
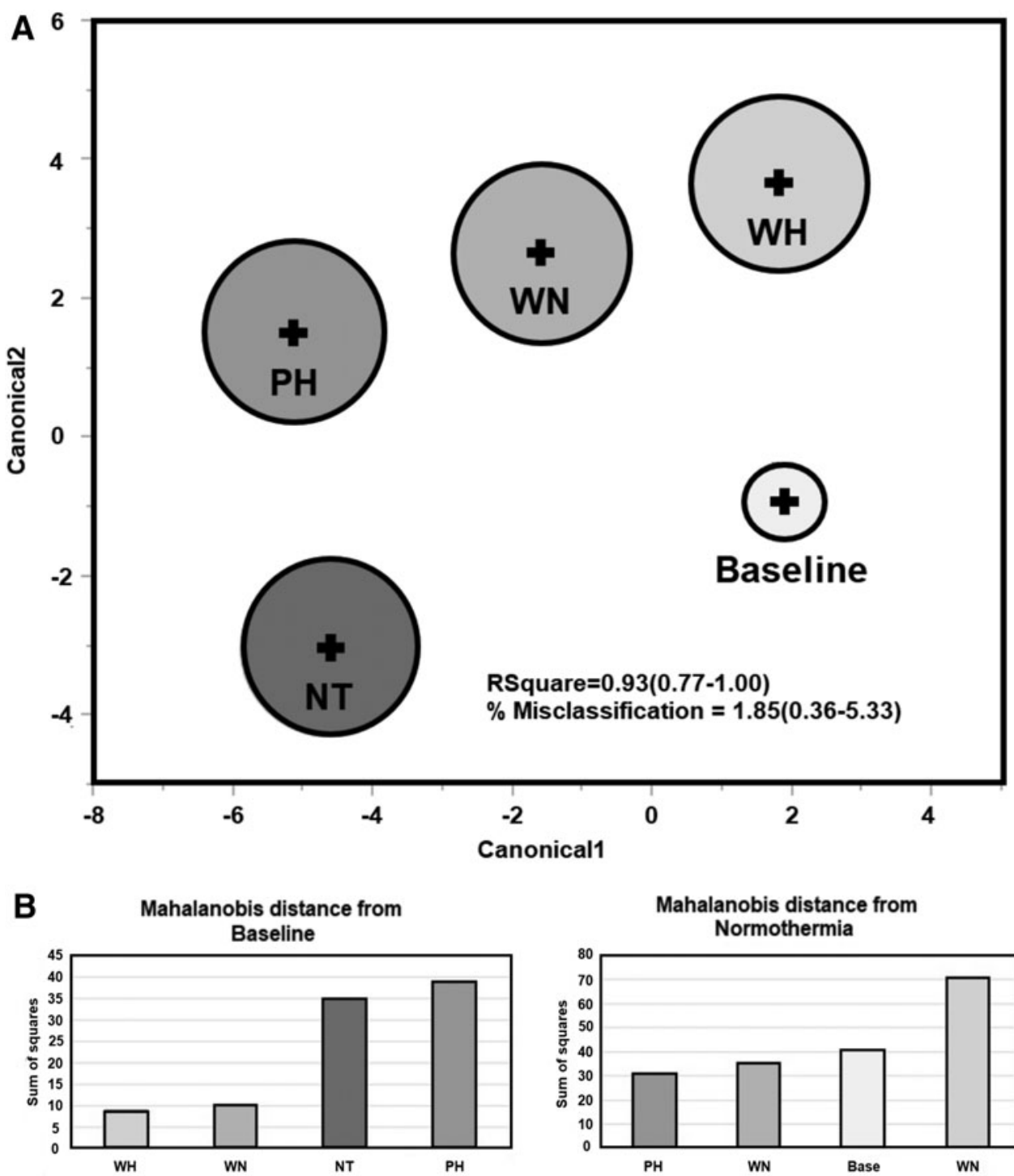

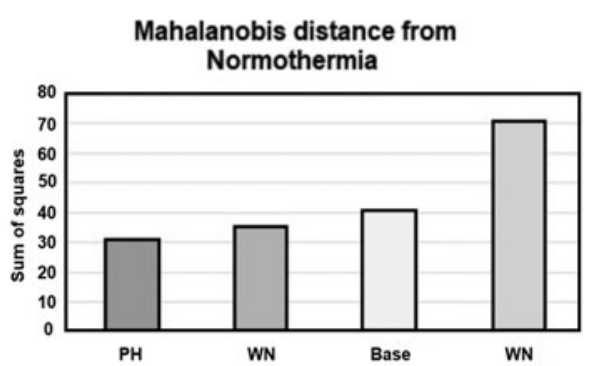

response that increases heat production up to $600 \%$ and triples oxygen consumption (Giesbrecht et al., 1994). Shivering resulting from physical cooling not only increases the difficulty of achieving and maintaining target temperature, but also early neurologic examination is compromised by the associated requirement for sedation and paralysis as well (Mahmood and Zweifler, 2007; Badjatia et al., 2008; Puttgen et al., 2009), which in turn can increase the possibility of ventilation-associated pneumonia (Lewis et al., 2014). In this study, intense shivering was only observed in physical cooling animals even though the degree of hypothermia was the same when compared with hypothermia induced by WIN55, 212-2.

Our previous studies (Sun et al., 2010; Weng et al., 2012; Ma et al., 2014) demonstrated that WIN55, 212-2 induced hypothermia and improved myocardial, cerebral function and duration of survival in a rat model of CA. This study confirmed the beneficial effects of WIN55, 212-2. In this study, decreased HR was observed in both $\mathrm{WH}$ and $\mathrm{WN}$ groups, while reduced MAP was found in $\mathrm{WH}, \mathrm{WN}$, and TH groups. Cardiovascular changes after $\mathrm{CB} 1$ and $\mathrm{CB} 2$ receptor activation include reduced $\mathrm{HR}$ and vasodilatation (Niederhoffer and Szabo, 1999; Niederhoffer et al., 2001; Padley et al., 2003). A significantly lower HR was observed in WH compared with other groups. This may result from induced hypothermia, which is consistent with our previous studies (Sun et al., 2010; Weng et al., 2012; Ma et al., 2014).

We demonstrated a low-density and inadequate perfused state of buccal microcirculation after resuscitation in all groups. However, in the WH and $\mathrm{PH}$ groups, there were significant improvements in PVD and MFI compared with NT and WN (PVD at PR1H, PVD, and MFI at PR4H). We demonstrated that microcirculation was significantly improved at PR4H WH group compared with $\mathrm{PH}$ group even with a significantly lower MAP, which may be the result of direct peripheral vasodilation after activation of CB1 receptors and the induction of hypothermia (Weng et al., 2012).

CA induces whole-body ischemia, causing multiple organ dysfunction and damage. Hypoxia causes an environment rich in reactive oxygen species, which leads to lipid peroxidation, inflammation, and subsequent lipase activation, with a concomitant increase in tissue lysophospholipid species (Chen et al., 2008). Several studies have demonstrated that lipids are 

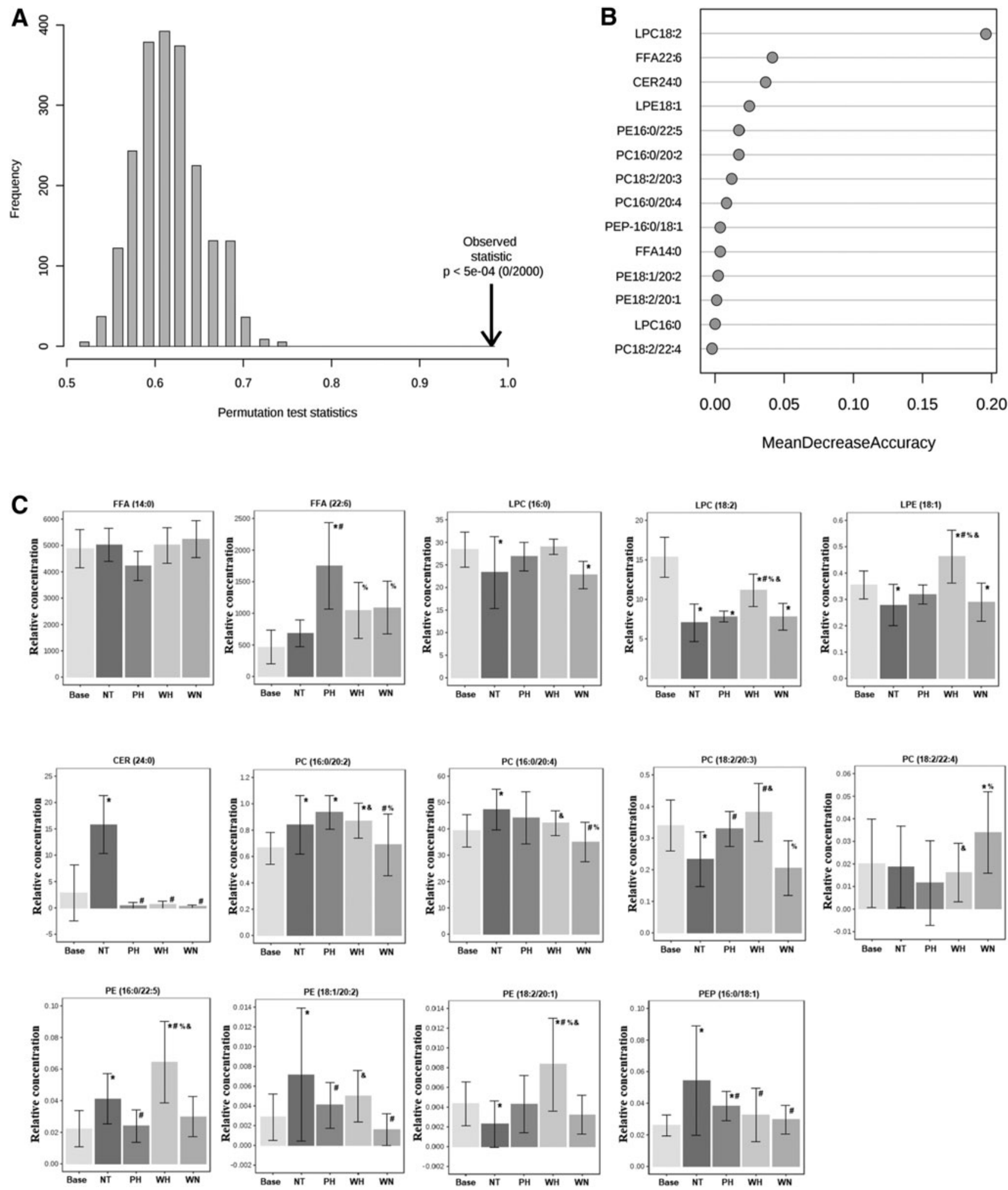

FIG. 7. (A) Permutation test indicates that the observed statistic is significantly different from 2000 random permutations ( $p=0.0005)$. (B) Random Forest method yields an error of 15\%. The mean decrease accuracy of a Random Forest validation shows that LPC 18:2, FFA 22:6, and CER 24:0 are more important variables in this model. (C) Group comparison of the three main predictors for group allocation. Analysis of variance with $p<0.05$. *Different from BL; " different from normothermia (NT); ${ }^{\circ}$ different from hypothermia $(\mathrm{PH})$; ${ }^{\&}$ different from WN. CER, ceramide; FFA, free fatty acid; LPC, lysophosphatidylcholine; PC, phosphatidylcholine; PE, phosphatidylethanolamine; PEP, phosphatidylethanolamine-plasmalogen. 
Table 2. Lipids Altered by Injury/Treatment

\begin{tabular}{|c|c|c|c|}
\hline Lipids & Biological function & Organ affected & References \\
\hline FFA (14:0) & $\begin{array}{l}\text { Myristic acid is a risk factor for cardiovascular mortality and } \\
\text { for negative outcome after severe trauma. It is also } \\
\text { involved in cellular stress in hepatic cells. }\end{array}$ & Heart and liver & $\begin{array}{l}\text { Ebbesson et al. }(2015) \\
\quad \text { Speziali et al. }(2018) \\
\text { Servià } \text { et al. }(2019)\end{array}$ \\
\hline FFA $(22: 6)$ & $\begin{array}{l}\text { DHA is essential for brain development and function. It has } \\
\text { neuroprotective function decreasing oxidative stress after } \\
\text { brain trauma. }\end{array}$ & Brain & $\underset{(2018)}{\text { Kim (2014); Zhu et al. }}$ \\
\hline LPC and LPE & $\begin{array}{l}\text { Animal study suggests that LPC and LPE accumulate in } \\
\text { ischemic heart and brain after cardiac arrest. It modulates } \\
\mathrm{Na}^{+} \text {channel in cardiac cells, slowing conduction. }\end{array}$ & Heart and brain & $\begin{array}{l}\text { Kinnaird et al. (1988); } \\
\quad \text { Shander et al. (1996) }\end{array}$ \\
\hline $\mathrm{PC}$ and $\mathrm{PE}$ & $\begin{array}{l}\text { Ischemia causes modulation of phosphatidylcholine and } \\
\text { phosphatidylethanolamine in the brain. Also these } \\
\text { phospholipids are the source of unsaturated fatty acids for } \\
\text { brain metabolism. }\end{array}$ & Brain & $\begin{array}{l}\text { Kim et al. (2015); } \\
\text { Sabogal-Guáqueta et al. } \\
\text { (2018) }\end{array}$ \\
\hline Plasmalogens & $\begin{array}{l}\text { Ceramides are generated in stress or inflammation and } \\
\text { mediate apoptosis. It is also produced in myocardium } \\
\text { after ischemia/reperfusion injury. }\end{array}$ & Brain & $\begin{array}{l}\text { Lessig and Fuchs (2009); } \\
\text { Dorninger et al. (2017) }\end{array}$ \\
\hline Ceramides & $\begin{array}{l}\text { Ceramides are generated in stress or inflammation and } \\
\text { mediate apoptosis. It is also produced in myocardium } \\
\text { after ischemia/reperfusion injury. }\end{array}$ & Heart & $\begin{array}{l}\text { Zhang et al. (2001); Yabu } \\
\quad \text { et al. (2015) }\end{array}$ \\
\hline
\end{tabular}

DHA, docosahexaenoic acid; FFA, free fatty acid; LPC, lysophosphatidylcholine; LPE, lysophosphatidylethanolamine; PC, phosphatidylcholine; PE, phosphatidylethanolamine.

altered by injury or treatment (Table 2). In this study, mass spectrometry-based lipidomic analysis of baseline lipid values and those for four different treatments were utilized to obtain a Linear Discriminant model that best represented the group's separation (Fig. 6). Baseline data (Base) from each group were binned together in a unique group representing the common start point for each of the four treatments. The analysis selected 14 lipids as predictors of group's membership for the six animals used in each treatment group. Group's separation based on canonical components showed that WH was the closest to baseline (Base), indicating that this treatment had the best metabolic outcome to bring back the animals to normal metabolism, which may be a protective mechanism of WH for outcomes of CPR.

The permutation test added decisive information for the existence of the model, showing that the observed statistics are significantly different from random permutation. Analysis showed that LPC 18:2, FFA 22:6, and CER 24:0 were the most important variables in the model. Docosahexaenoic acid (FFA 22:6; DHA) is an omega-3 fatty acid found in high concentrations in the brain as a constituent of neuronal membranes in gray matter, white matter, and glial cells. DHA treatment combined with hypothermia in a posthypoxia-ischemia rat's model has shown sustained improvement and reduced brain damage (Berman et al., 2013). In a review of thermogenesis regulation in brown adipose tissue (BAT), Kuroshima et al. (2001) suggested that DHA levels are closely associated with BAT thermogenesis, and an increase in DHA enhances BAT thermogenesis.

Activation of thermogenesis in response to hypothermia could be deleterious to the brain in conditions such as CA (Tupone et al., 2016). Previous studies (Belayev et al., 2009; Eady et al., 2013, 2014; Hong et al., 2014) showed that FFA 22:6 (DHA) treatment group in rodent models of brain ischemia had improved neurological and histological outcomes, accompanied by decreases in blood-brain barrier disruption, brain edema, malondialdehyde production, inflammatory cell infiltration, interleukin-6 expression, and caspase-3 activity (Pan et al., 2009). Muldoon et al. (2010) also found that DHA was associated with enhanced cognition in humans. Therefore, it is interesting to note that despite an increase in DHA and possible thermogenic activation in the PH group as a marker of hypothermia only treatment, both groups receiving WIN55, 212-2 did not show significant elevation of DHA. Therefore, pharmacological hypothermia induced by WIN55, 212-2 in combination with physical hypothermia may adjust its balance and acquire more benefits, which we are exploring further.

Ceramide has been described as a second messenger. All of these specimens control cell proliferation, differentiation, and apoptosis. Hypothermia or $\beta$-adrenergic agonists selectively reduce adipose ceramides (Chaurasia et al., 2016). As shown in Figure 7C, the ceramide concentration increased in NT compared with Base, while it decreased in $\mathrm{WH}, \mathrm{WN}$, and $\mathrm{PH}$. We also demonstrated a drop in $\mathrm{WH}$, which may be the result of $\mathrm{CB}$ receptor activation and induction of hypothermia. It has been shown that exogenous ceramides could induce hypothalamic lipotoxicity, ER stress, and decreased sympathetic tone to BAT, which leads to decreased thermogenesis (Contreras et al., 2014). Ceramide effects on adipose metabolism are cell autonomous. Ceramides regulate the activation of volume-sensitive chloride current in cardiomyocytes, which controls cardiac electromechanical activity and cell volume as well as apoptosis and contributes to lipotoxic cardiomyopathy (Raucci et al., 2010). Distinct plasma ceramide ratios are significant predictors of cardiovascular death in patients with stable coronary artery disease and acute coronary syndromes, over and above currently used lipid markers (Laaksonen et al., 2016).

It has been demonstrated that simple sphingolipids, particularly ceramide, appear to be involved in the regulation of specific aspects of neuronal proliferation, differentiation, survival, and apoptosis (Buccoliero et al., 2003). Recently, cannabinoids 
have been shown to protect astrocytes from ceramide-induced apoptosis through the phosphatidylinositol 3-kinase/protein kinase B pathway (Gómez Del Pulgar et al., 2002).

Elevation of LPC 18:2 level compared with NT was the more relevant lipid modulation in WH. Its decrease in plasma concentration in NT could indicate accumulation in the ischemic myocardium. The accumulation of lysophosphatidylcholine species occurs very fast, and is described in ischemic hearts in rats and other species (Daleau, 1999). Lysophosphatidylcholine toxicity to the myocardium induces electrophysiological changes, including action potential alterations and cardiac arrhythmias (Yu et al., 1998). Although LPCs are commonly seen as proinflammatory and proatherogenic metabolites (Schmitz and Ruebsaamen, 2010), recent population-based studies have demonstrated a protective effect of LPCs on cardiovascular risk, which is associated with a decreased risk of incident myocardial infarction (Ganna et al., 2014) and thus has a negative association with coronary heart disease (Ward-Caviness et al., 2017). The relative resaturation of LPC 18:2 to baseline levels with WH suggests cardiac protection, which confirms and extends these previous findings.

\section{Limitations}

There are some limitations for this study: (1) The endpoint of our study was performed after ROSC 4 hours. (2) Lipid metabolism analysis only measured plasma concentration. Further research regarding mechanism will also investigate the lipid changes that accompany CA and treatments in tissue (liver, kidney, heart, and brain).

\section{Conclusion}

Hypothermia after resuscitation reduces the severity of postresuscitation myocardial and microcirculatory dysfunction. WIN55-212-2 produced the same degree of hypothermia as physical cooling without the disadvantage of shivering. WIN55-212-2 with hypothermia preserved lipid metabolism, which may be a protective mechanism after ischemia and reperfusion. Meanwhile, LPC 18:2, FFA 22:6, and CER (24:0) may be important predictors of outcomes of CA. Our study indicates that early and effective hypothermia after CA may be induced by pharmacological means alone or in combination with physical hypothermia.

\section{Author Disclosure Statement}

No competing financial interests exist.

\section{Funding Information}

This study was funded in part by the project of Leading Talents in Pearl River Talent Plan of Guangdong Province, China (No. 81000-42020004) and People's Livelihood Science and technology_-Basic Research on medical and health application of Soochow, Jiangsu Province, China (No. SYS20 19075). Research reported in this publication was also supported by research grants from National Institutes of Health under grant numbers HD087198 (to D.S.W.). The content is solely the responsibility of the authors and does not necessarily represent the official views of the National Institutes of Health.
This work also received support through Zoll foundation and a Young Investigator Award from SCIEX for clinical lipidomic research (D.S.W.).

\section{References}

Badjatia N, Sronglilis E, Prescutti M, et al. Metabolic impact of shivering during therapeutic temperature modulation: the bedside shivering assessment scale. Stroke 2008;39:32423247.

Belayev L, Khoutorova L, Atkins KD, et al. Robust docosahexaenoic acid-mediated neuroprotection in a rat model of transient, focal cerebral ischemia. Stroke 2009;40:31213126.

Berman DR, Mozurkewich E, Liu Y, et al. Docosahexaenoic acid augments hypothermic neuroprotection in a neonatal rat asphyxia model. Neonatology 2013;104:71-78.

Bernard SA, Gray TW, Buist MD, et al. Treatment of comatose survivors of out-of-hospital cardiac arrest with induced hypothermia. N Engl J Med 2002;346:557-563.

Buccoliero R, Futerman AH. The roles of ceramide and complex sphingolipids in neuronal cell function. Pharmacol Res 2003;47:409-419.

Callaway CW, Donnino MW, Fink EL, et al. Part 8: Post-cardiac arrest care 2015 American Heart Association Guidelines update for cardiopulmonary resuscitation and emergency cardiovascular care. Circulation 2015;132:S465-S482.

Chaurasia B, Kaddai VA, Lancaster GI, et al. Adipocyte ceramides regulate subcutaneous adipose browning, inflammation, and metabolism. Cell Metab 2016;24:820-834.

Chen ZH, Saito Y, Yoshida Y, et al. Effect of oxygen concentration on free radical-induced cytotoxicity. Biosci Biotechnol Biochem 2008;72:1491-1497.

Contreras C, González-García I, Martínez-Sánchez N, et al. Central ceramide-induced hypothalamic lipotoxicity and ER stress regulate energy balance. Cell Rep 2014;9:366-377.

Daleau P. Lysophosphatidylcholine, a metabolite which accumulates early in myocardium during ischemia, reduces gap junctional coupling in cardiac cells. Mol Cell Cardiol 1999; 31:1391-401.

De Backer D, Hollenberg S, Boerma C, et al. How to evaluate the microcirculation: report of a round table conference. Crit Care 2007;11:R101.

De Witte J, Sessler DI. Perioperative shivering: physiology and pharmacology. Anesthesiology 2002;96:467-484.

Dorninger F, Forss-Petter S, Berger J. From peroxisomal disorders to common neurodegenerative diseases - the role of ether phospholipids in the nervous system. FEBS Lett 2017; 591:2761-2788.

Eady TN, Khoutorova L, Anzola DV, et al. Acute treatment with docosahexaenoic acid complexed to albumin reduces injury after a permanent focal cerebral ischemia in rats. PLoS One 2013;8:e77237.

Eady TN, Khoutorova L, Obenaus A, et al. Docosahexaenoic acid complexed to albumin provides neuroprotection after experimental stroke in aged rats. Neurobiol Dis 2014;62: $1-7$.

Ebbesson SOE, Voruganti VS, Higgins PB, et al. Fatty acids linked to cardiovascular mortality are associated with risk factors. Int $\mathrm{J}$ Circumpolar Health 2015;74:28055.

Fink EL, Callaway CW, Tisherman SA, et al. Winning the cold war: inroads into implementation of mild hypothermia after cardiac arrest in adults from the European Resuscitation Council Hypothermia After Cardiac Arrest Registry Study Group. Crit Care Med 2007;35:1199-1202. 
Gaieski DF, Band RA, Abella BS, et al. Early goal-directed hemodynamic optimization combined with therapeutic hypothermia in comatose survivors of out-of-hospital cardiac arrest. Resuscitation 2009;80:418-424.

Ganna A, Salihovic S, Sundström J, et al. Large-scale metabolomic profiling identifies novel biomarkers for incident coronary heart disease. PLoS Genet 2014;10:e1004801.

Giesbrecht GG, Sessler DI, Mekjavic IB, et al. Treatment of mild immersion hypothermia by direct body-to-body contact. J Appl Physiol 1994;76:2373-2379.

Gómez Del Pulgar T, De Ceballos ML, Guzmán M, et al. Cannabinoids protect astrocytes from ceramide-induced apoptosis through the phosphatidylinositol 3-kinase/protein kinase B pathway. J Biol Chem 2002;277:36527-36533.

Hong SH, Belayev L, Khoutorova L, et al. Docosahexaenoic acid confers enduring neuroprotection in experimental stroke. J Neurol Sci 2014;338:135-141.

Hypothermia after Cardiac Arrest Study Group. Mild therapeutic hypothermia to improve the neurologic outcome after cardiac arrest. N Engl J Med 2002;346:549-556.

Kim H-Y. Neuroprotection by docosahexaenoic acid in brain injury. Mil Med 2014;179:106-111.

Kim J, Lampe JW, Yin T, et al. Phospholipid alterations in the brain and heart in a rat model of asphyxia-induced cardiac arrest and cardiopulmonary bypass resuscitation. Mol Cell Biochem 2015;408:273-281.

Kinnaird AA, Choy PC, Man RY. Lysophosphatidylcholine accumulation in the ischemic canine heart. Lipids 1988;23:32-35.

Kuroshima A, Ohinata H, Kikuchi-Utsumi K, et al. Brown adipose tissue and nonshivering thermogenesis in stressful states. In: Kosaka M, Sugahara T, Schmidt KL, Simon E, eds. Thermotherapy for Neoplasia, Inflammation, and Pain. Tokyo, Japan: Springer, 2001, pp. 118-126.

Laaksonen R, Ekroos K, Sysi-Aho M, et al. Plasma ceramides predict cardiovascular death in patients with stable coronary artery disease and acute coronary syndromes beyond LDLcholesterol. Eur Heart J 2016;37:1967-1976.

Lampe JW, Becker LB. Rapid cooling for saving lives: a bioengineering opportunity. Epert Rev Med Devices 2007;4:441-446.

Lessig J, Fuchs B. Plasmalogens in biological systems: their role in oxidative processes in biological membranes, their contribution to pathological processes and aging and plasmalogen analysis. Curr Med Chem 2009;16:2021-2041.

Lewis SC, Li L, Murphy MV, et al. CDC Prevention Epicenters. Risk factors for ventilator-associated events: a case-control multivariable analysis. Crit Care Med 2014;42:1839-1848.

Lopez M, Sessler DI, Walter K, et al. Rate and gender dependence of the sweating, vasoconstriction, and shivering thresholds in humans. Anesthesiology 1994;80:780-788.

Ma L, Lu X, Xu J, et al. Improved cardiac and neurologic outcomes with postresuscitation infusion of cannabinoid receptor agonist WIN55, 212-2 depend on hypothermia in a rat model of cardiac arrest. Crit Care Med 2014;42:e42-e48.

MacLaren R, Gallagher J, Shin J, et al. Assessment of adverse events and predictors of neurological recovery after therapeutic hypothermia. Ann Pharmacother 2014;48:17-25.

Mahmood MA, Zweifler RM. Progress in shivering control. J Neurol Sci 2007;261:47-54.

Mongardon N, Dumas F, Ricome S, et al. Postcardiac arrest syndrome: from immediate resuscitation to long-term outcome. Ann Intensive Care 2011;1:45.

Muldoon MF, Ryan CM, Sheu L, et al. Serum phospholipid docosahexaenoic acid is asssociated with cognitive functioning during middle adulthood. J Nutr 2010;140:848-853.
Murakami M. Lipid mediators in life science. Exp Anim 2011; 60:7-20.

Niederhoffer N, Hansen HH, Fernandez-Ruiz JJ, et al. Effects of cannabinoidson adrenaline release from adrenal medullary cells. Br J Pharmacol 2001;134:1319-1327.

Niederhoffer N, Szabo B. Effect of the cannabinoid receptor agonist WIN55212-2 on sympathetic cardiovascular regulation. Br J Pharmacol 1999;126:457-466.

Padley JR, Li Q, Pilowsky PM, et al. Cannabinoid receptor activation in the rostral ventrolateral medulla oblongata evokes cardiorespiratory effects in anaesthetised rats. Br J Pharmacol 2003;140:384-394.

Pan HC, Kao TK, Ou YC, et al. Protective effect of docosahexaenoic acid against brain injury in ischemic rats. J Nutr Biochem 2009;20:715-725.

Pollock JS, Hollenbeck RD, Wang L, et al. Delirium in survivors of cardiac arrest treated with mild therapeutic hypothermia. Am J Crit Care 2016;25:e81-89.

Puttgen HA, Pantle H, Geocadin RG. Management of cardiac arrest patients to maximize neurologic outcome. Curr Opin Crit Care 2009;15:118-124.

Raucci FJ, Wijesinghe DS, Chalfant CE, et al. Exogenous and endogenous ceramides elicit volume-sensitive chloride current in ventricular myocytes. Cardiovasc Res 2010;86:55-62.

Rink C, Khanna S. Significance of brain tissue oxygenationand the arachidonic acid cascade in stroke. Antioxid Redox Signal 2011;14:1889-1903.

Sabogal-Guáqueta AM, Villamil-Ortiz JG, Arias-Londoño JD, et al. Inverse phosphatidylcholine/phosphatidylinositol levels as peripheral biomarkers and phosphatidylcholine/lysophosphati dylethanolamine-phosphatidylserine as hippocampal indicator of postischemic cognitive impairment in rats. Front Neurosci 2018;12:989.

Salgado DR, He X, Su F, et al. Sublingual microcirculatory effects of enalaprilat in an ovine model of septic shock. Shock 2011;35:542-549.

Schmitz G, Ruebsaamen K. Metabolism and atherogenic disease association of lysophosphatidylcholine. Atherosclerosis 2010;208:10-18.

Servià L, Jové M, Sol J, et al. A prospective pilot study using metabolomics discloses specific fatty acid, catecholamine and tryptophan metabolic pathways as possible predictors for a negative outcome after severe trauma. Scand J Trauma Resusc Emerg Med 2019;27:56.

Shander GS, Undrovinas AI, Makielski JC. Rapid onset of lysophosphatidylcholine-induced modification of whole cell cardiac sodium current kinetics. J Mol Cell Cardiol 1996;28: 743-753.

Speziali G, Liesinger L, Gindlhuber J, et al. Myristic acid induces proteomic and secretomic changes associated with steatosis, cytoskeleton remodeling, endoplasmic reticulum stress, protein turnover and exosome release in HepG2 cells. J Proteomics 2018;181:118-130.

Stub D, Bernard S, Duffy SJ, et al. Post cardiac arrest syndrome: a review of therapeutic strategies. Circulation 2011; 123:1428-1435.

Sun S, Tang W, Song F, et al. Pharmacologically induced hypothermia with cannabinoid receptor agonist WIN55, 212-2 after cardiopulmonary resuscitation. Crit Care Med 2010;38: 2282-2286.

Tupone D, Cetas JS, Morrison SF. Hibernation, hypothermia and a possible therapeutic "shifted homeostasis" induced by central activation of A1 adenosine receptor (A1AR). Nihon Shinkei Seishin Yakurigaku Zasshi 2016;36:51-54. 
Ward-Caviness CK, Xu T, Aspelund T, et al. Improvement of myocardial infarction risk prediction via inflammation-associated metabolite biomarkers. Heart 2017;103:1278-1285.

Weng Y, Sun S, Park J, et al. Cannabinoid 1 (CB1) receptor mediates WIN55, 212-2 induced hypothermia and improved survival in a rat post-cardiac arrest model. Resuscitation 2012; 83:1145-1151.

Yabu T, Shiba H, Shibasaki Y, et al. Stress-induced ceramide generation and apoptosis via the phosphorylation and activation of nSMase1 by JNK signaling. Cell Death Differ 2015; 22:258-273.

Yoshida K, Li X, Cano G, et al. Parallel preoptic pathways for thermoregulation. J Neurosci 2009;29:11954-11964.

Yu, L, Netticadan, T, Xu, YJ. et al. Mechanisms of lysophosphati dylcholine-induced increase in intracellular calcium in rat cardiomyocytes. J Pharmacol Exp Ther 1998;286:1-8.

Zhang DX, Fryer RM, Hsu AK, et al. Production and metabolism of ceramide in normal and ischemicreperfused myocardium of rats. Basic Res Cardiol 2001;96: 267-274.

Zhu W, Ding Y, Kong W, et al. Docosahexaenoic acid (DHA) provides neuroprotection in traumatic brain injury models via activating Nrf2-ARE signaling. Inflammation 2018;41: 1182-1193.
Address correspondence to:

Wanchun Tang, MD

Department of Emergency Medicine VCU School of Medicine

Box 980266

Sanger Hall, 1101 E. Marshall Street

Richmond, VA 23298-0279

USA

E-mail: wanchun.tang@vcuhealth.org

Dayanjan S. Wijesinghe, PhD

Department of Pharmacotherapy and Outcomes Sciences

School of Pharmacy

Virginia Commonwealth University

Box 980533

Robert Blackwell Smith Bldg. Rm. 652A

410 North 12th Street

Richmond, VA 23298-5048

USA

E-mail: wijesingheds@vcu.edu 